\title{
LIBERAÇÃO DE CO 2 , BIOMASSA MICROBIANA E FÓSFORO DISPONÍVEL EM SOLO ADICIONADO DE MATÉRIA SECA DE POAIA-BRANCA ${ }^{1}$
}

\author{
ELIETE S. MACHADO ${ }^{2}$, MARLI T.A. MINHONI ${ }^{3}$ e LEONARDO T. BÜLL ${ }^{4}$
}

\section{RESUMO}

O presente experimento, inteiramente casualizado, foi desenvolvido em condições de laboratório no Departamento de Defesa Fitossanitária, FCA/UNESP - Botucatu, entre julho e setembro de 1992. Amostras de Areia Quartzosa equivalentes à $40 \mathrm{~g}$ de terra seca à $105^{\circ} \mathrm{C} \pm 2$ com ou sem adição de $1,9 \mathrm{~g}$ de matéria seca de plantas de poaia-branca (Richardia brasiliensis), 0,19 g de nitrogênio $\left(\mathrm{NH}_{4}\right)_{2} \mathrm{SO}_{4} \mathrm{e}$ $0,88 \mathrm{~g}$ de apatita de Araxá, foram incubadas no escuro a $25^{\circ} \mathrm{C} \pm 2$, com umidade mantida a $60 \%$ da capacidade de retenção de água. Durante a incubação, determinou-se $\mathrm{o} \quad \mathrm{CO}_{2}$ liberado, utilizando-se o método de retenção em $\mathrm{NAOH}$ seguida de titulometria com $\mathrm{HCl}$; a biomassa microbiana, método de fumigação-incubação; o pH e a quantidade de fósforo extraído por resina. A maior liberação de $\mathrm{CO}_{2}$ ocorreu durante os dez primeiros dias de incubação, com $77 \%$ do total de carbono liberado nos tratamentos com adição de poaia, e $37 \%$ nos tratamentos sem adição da mesma. A liberação de $\mathrm{CO}_{2}$ foi 57 vezes maior nos tratamentos com poaia em relação ao controle. A poaia também provocou aumentos na biomassa microbiana (média de 8 vezes a biomassa do tratamento controle), e a adição de nitrogênio e/ou fosfato de rocha junto à poaia antecipou os picos de formação de biomassa de 20 para 10 dias de incubação. Os níveis de fósforo disponível foram maiores no tratamento com adição de fosfato de rocha apenas. A poaia também alcalinizou o sistema, não permitindo desta forma, observar-se relação significativa entre $\mathrm{pH}$ e teor de fósforo disponível.

Palavras chave: Fosfato de rocha, planta daninha, Richardia brasiliensis.

\section{ABSTRACT \\ $\mathrm{CO}_{2}$ liberation, microbial biomass and available phosphorus in soil supplemented with Brasil callality dry matter}

The present experiment, totally randomized, was carried out under laboratory conditions at the Departamento de Defesa Fitossanitária, FCA/UNESP-Botucatu, between July and September, 1992. Fresh air-dry samples of quartz sand representing $40 \mathrm{~g}$ of dry soil each, at $105^{\circ} \mathrm{C} \pm 2$, with or without addition of $1.90 \mathrm{~g}$ of dry matter of "poaia-branca" (Richardia brasiliensis) plants, $0.19 \mathrm{~g}$ of nitrogen $\left(\mathrm{NH}_{4}\right)_{2} \mathrm{SO}_{4}$ and $0.88 \mathrm{~g}$ of Araxá apatite, were incubated under dark conditions at $25{ }^{\circ} \mathrm{C} \pm 2$, with moisture held at $60 \%$ water retention capacity. During incubation, $\mathrm{CO}_{2}$-liberation was determined by means of the method of retention in $\mathrm{NaOH}$ followed by titration with $\mathrm{HCl}$; microbial biomass was determined through the fumigation-incubation method, while $\mathrm{pH}$ and phosphorus extract by resin. $\mathrm{CO}_{2}$-liberation was high during the first ten days of incubation, with $77 \%$ of total carbon released in treatments with "poaia" addition, and $37 \%$ in treatments without addition. $\mathrm{CO}_{2}-$ liberation was 57 times higher in the "Poaia" treatments compared to the control.

\footnotetext{
${ }^{1}$ Recebido para publicação em 02/10/97 e na forma revisada em 25/11/98. Apoio financeiro da CAPES.

2 Bióloga, aluna do Curso de Pós -graduação em Proteção de Plantas, Dept ${ }^{\circ}$ de Defesa Fitossanitária, FCA/UNESP. C.P. 237, CEP 18.603-970, Botucatu/SP. Bolsista da CAPES.

${ }^{3}$ Professora Assistente Doutora do Deptartamento de Defesa Fitossanitária, FCA/UNESP, Botucatu/SP.

${ }^{4}$ Professor Adjunto do Departamento de Ciência do Solo, FCA/UNESP, Botucatu/SP. Bolsista do CNPq.
} 
"Poaia"also promoted increases in microbial biomass (an average of 8 times the control biomass), and nitrogen and/or rock phosphate added to "poaia" anticipated peak biomass formation from the $20^{\text {th }}$ to the $10^{\text {th }}$ day of incubation. Levels of available phosphorus were higher in the rock-phosphate supplemented

\section{INTRODUÇÃO}

As plantas daninhas, competidoras eficientes por água, luz, e nutrientes, diminuem a produtividade de certas culturas. Ademais, podem intervir diretamente no crescimento destas culturas e na comunidade microbiana através de inibição química, conhecida como alelopatia. $\mathrm{O}$ processo envolve compostos secundários liberados pela planta, com ou sem subseqüente degradação enzimática, realizada por microrganismos (Alves, 1992).

Richardia brasiliensis, conhecida como poaia-branca, poaia-do-campo ou simplesmente poaia, tem sido descrita como planta daninha. Efeitos inibitórios drásticos de Richardia brasiliensis foram verificados na germinação e desenvolvimento de pepino e arroz (Salvati, 1988).

$\begin{array}{rrr}\text { A utilização de } & \text { fosfatos de rocha na } \\ \text { agricultura tornou-se } & \text { uma alternativa }\end{array}$ economicamente importante. Entretanto, sua eficiência depende da solubilização e velocidade de dissolução, uma vez que as plantas absorvem fósforo quase que exclusivamente da solução do solo. Desta forma, o fosfato insolúvel presente e/ou adicionado ao solo, deve tornar-se solúvel antes de ser absorvido. Neste contexto, a solubilização microbiana de fosfatos de rocha é reconhecida há muitos anos e tem sido manejada com o intuito de melhorar o aproveitamento dos fosfatos naturais aplicados ao solo. A adição de matéria orgânica, estimulando a comunidade microbiana, tem resultado em aumentos do fósforo disponível, evidência da solubilização microbiana (Sperber, 1958; Minhoni et al., 1991; Eira, 1992 e Minhoni et al., 1996).

Sendo assim, os objetivos deste trabalho foram verificar os efeitos da matéria seca de poaia-branca (Richardia brasiliensis) sobre a comunidade microbiana do solo, em termos de treatments only. "Poaia" alkalinized the system, which made it impossible to observe any significant relation between $\mathrm{pH}$ and the available phosphorus content.

Key words: Rock phosphate, available phosphorus, weed, Richardia brasiliensis.

$\mathrm{CO}_{2}$ liberado, formação de biomassa microbiana, solubilização de fosfato de rocha e alterações no $\mathrm{pH}$ do solo.

\section{MATERIAL E MÉTODOS}

$\mathrm{O}$ experimento, inteiramente casualizado, foi conduzido em laboratório, no Departamento de Defesa Fitossanitária FCA/UNESP - Botucatu/SP, entre julho e setembro de 1992, e constou da incubação de amostras de solo adicionadas de matéria seca de poaia-branca, nitrogênio e/ou fosfato de rocha, seguida da quantificação de carbono liberado do solo, biomassa microbiana, fósforo disponível e $\mathrm{pH}$.

Os tratamentos, com quatro repetições cada, foram: controle (sem adição de poaia, nitrogênio e/ou fosfato de rocha); poaia (com adição de poaia apenas); poaia+N (com adição de poaia e nitrogênio); poaia+N+FR (com adição de poaia, nitrogênio e fosfato de rocha); poaia $+\mathrm{FR}$ (com adição de poaia e fosfato de rocha); e FR (com adição de fosfato de rocha).

Amostras superficiais $(0-20 \mathrm{~cm})$ de Areia Quartzosa do município de Anhembi (SP) foram secas ao ar, passadas em peneira de malha $2 \mathrm{~mm}$ e analisadas, apresentando $823 \mathrm{~g} \mathrm{~kg}^{-1}$ de areia, $135 \mathrm{~g} \mathrm{~kg}^{-1}$ de silte e $42 \mathrm{~g} \mathrm{~kg}^{-1}$ de argila em sua composição granulométrica; $\mathrm{pH}$ em $\mathrm{CaCl}_{2}: 3,9 ; \mathrm{C}$ via seca: $3,05 \mathrm{~g} \mathrm{~kg}^{-1}$; $\mathrm{N}$ total: $0,5 \mathrm{~g} \mathrm{~kg}^{-1}$; matéria orgânica: $13 \mathrm{~g} \mathrm{~kg}^{-1} ; \quad \mathrm{P}: 6 \mathrm{mg} \mathrm{kg}{ }^{-1} ; \mathrm{H}^{+}+\mathrm{Al}^{\beta^{+}}$: 34 mmol $_{\mathrm{c}} \quad \mathrm{kg}^{-1} ; \quad \mathrm{K}^{+}: 1,4 \quad \mathrm{mmol}_{\mathrm{c}} \mathrm{kg}^{-1} ; \quad \mathrm{Ca}^{2+}$ : 4,0 $\mathrm{mmol}_{\mathrm{c}} \mathrm{kg}^{-1} ; \mathrm{Mg}^{2+}: 3,0 \mathrm{mmol}_{\mathrm{c}} \mathrm{kg}^{-1} \mathrm{e} \mathrm{V}: 19 \%$.

Plantas inteiras de poaia-branca foram coletadas em diversas regiões do município de Botucatu (SP), durante o período de um ano. Após 
as coletas, as plantas foram lavadas em água corrente, secas em estufa com circulação forçada de ar à $\pm 30^{\circ} \mathrm{C}$ e moídas em moinho de aço inoxidável. A análise química deste material revelou $359 \mathrm{~g} \mathrm{~kg}^{-1}$ de C total (via seca) e $21 \mathrm{~g} \mathrm{~kg}^{-1}$ de $\mathrm{N}$.

Conforme os tratamentos, amostras de solo equivalentes a $40 \mathrm{~g}$ de solo seco à $105^{\circ} \mathrm{C} \pm 2$ foram adicionadas de $1,89 \mathrm{~g}$ de matéria seca moída de poaia-branca, $0,19 \mathrm{~g}$ de sulfato de amônio (complementação nitrogenada) e $0,88 \mathrm{~g}$ de apatita de Araxá (contendo $310 \mathrm{~g} \mathrm{~kg}^{-1}$ de $_{2} \mathrm{O}_{5}$ ). As amostras foram homogeneizadas, dispostas em frascos de vidro com $28,27 \mathrm{~cm}^{2}$ de área e $4 \mathrm{~cm}$ de altura, e umedecidas à $60 \%$ da capacidade de retenção de água (c.r.a.) pela adição de $4,8 \mathrm{ml}$ de água destilada. Após o preparo, as parcelas foram incubadas no escuro a $25{ }^{\circ} \mathrm{C}{ }^{ \pm} 2$ com umidade corrigida periodicamente para $60 \%$ da c. r. a., com base no peso das amostras.

Para quantificação do $\mathrm{CO}_{2}$ liberado, quatro parcelas de cada tratamento foram colocadas, individualmente, em potes de vidro transparente, boca larga, tampa rosqueável, capacidade 3,6 L, contendo cerca de $5 \mathrm{ml}$ de água destilada na base, para se manter a atmosfera úmida. Juntamente com as amostras colocou-se, também, um frasco contendo $20 \mathrm{ml}$ de $\mathrm{NaOH}$ $1 \mathrm{~mol} \mathrm{~L} \mathrm{~L}^{-1}$, para retenção do $\mathrm{CO}_{2}$ liberado. Foram preparados também três potes contendo apenas o frasco com $20 \mathrm{ml} \mathrm{de} \mathrm{NaOH} 1 \mathrm{~mol} \mathrm{~L}^{-1}$; tais parcelas constituíram-se nas provas em branco. Todos os potes foram tampados e incubados no escuro à temperatura de $25 \pm 2{ }^{\circ} \mathrm{C}$. Aos $2,5,7,10,15,20$, $25,30,35,40,45,50,55$ e 60 dias de incubação, o $\mathrm{NaOH}$ foi retirado e adicionado de $5 \mathrm{ml}$ de solução aquosa saturada de $\mathrm{BaCl}_{2}$ para precipitação do $\mathrm{Na}_{2} \mathrm{CO}_{3}$ formado. A seguir, foram adicionadas três gotas de solução alcoólica a $10 \mathrm{~g}$ $\mathrm{kg}^{-1}$ de fenolftaleína como indicador do ponto de viragem de rosa para branco leitoso e procedeu-se a titulometria com $\mathrm{HCl} 0,65 \mathrm{~mol} \mathrm{~L}^{-1}$. Após a retirada do $\mathrm{NaOH}$, novos frascos contendo $20 \mathrm{ml}$ de $\mathrm{NaOH} 1 \mathrm{~mol} \mathrm{~L}^{-1}$ foram colocados nos potes de vidro, e os mesmos reincubados.
$\mathrm{O} \mathrm{CO}_{2}$ liberado da amostra foi calculado utilizando-se a fórmula:

$\mathrm{mg} \mathrm{C}-\mathrm{CO}_{2} \mathrm{~kg}^{-1}$ solo $=[\mathrm{HCl}(\mathrm{ml})$ branco $-\mathrm{HCl}$ (ml) parcela] . 6. NHCl/ 40

Para a quantificação da biomassa microbiana do solo, adotou-se o método proposto por Jenkinson \& Powlson (1976). Aos 0, 5, 10, 20, 30 e 40 dias, considerando-se cada um dos tratamentos, quatro parcelas foram fumigadas com clorofórmio isento de álcool, durante cerca de 24 horas. Em seguida procedeu-se a defumigação e inoculação das parcelas com $2 \mathrm{ml}$ de extrato de solo $\left(50 \mathrm{ml}\right.$ solo/400 $\mathrm{ml} \mathrm{H} \mathrm{H}_{2} \mathrm{O}$ ), para reposição da comunidade microbiana. Após, adotou-se procedimento descrito para avaliação de liberação de $\mathrm{CO}_{2}$, com incubação das parcelas fumigadas e mais quatro parcelas não fumigadas de cada tratamento.

A biomassa microbiana foi calculada através da equação proposta por Jenkinson \& Powlson (1976): $\mathrm{B}=\mathrm{X}-\mathrm{Y} / \mathrm{k}$ onde:

B: biomassa microbiana, $\mathrm{mgC}-\mathrm{CO}_{2} \mathrm{~kg}^{-1} \mathrm{de}$ solo;

$\mathrm{X}$ : carbono liberado de parcela fumigada, mgC- $\mathrm{CO}_{2} \mathrm{~kg}^{-1}$ solo, dentro de um período de 10 dias;

Y: carbono liberado de parcela não fumigada, $\mathrm{mgC}-\mathrm{CO}_{2} \mathrm{~kg}^{-1}$ solo, dentro do mesmo período de 10 dias.

$\mathrm{O}$ fator $\mathrm{K}$, que representa a fração de carbono da biomassa mineralizada para $\mathrm{CO}_{2}$ durante o período de incubação, foi adotado como 0,45, conforme Bonde et al., (1989), Saffigna et al., (1989), Couteaux et al., (1989).

Em onze épocas de incubação $(0,2,3,5$, $7,10,20,30,40,50$ e 60 dias), quatro parcelas de cada tratamento foram secas ao ar, homogeneizadas e submetidas à análise de fósforo disponível e pH, no Departamento de Ciência do Solo da FCA - UNESP - Botucatu/SP, segundo metodologia descrita por Raij \& Quaggio (1983).

Os dados obtidos foram submetidos à análise de variância, com teste de Tukey a $5 \%$ de significância, para comparação entre as médias (Snedecor \& Cochran, 1972). 


\section{RESULTADOS E DISCUSSÃO}

Resultados referentes à taxa diária média de carbono liberado do solo são apresentados na Tabela 1. Para todos os tratamentos, a maior liberação de $\mathrm{CO}_{2}$ ocorreu durante os dez primeiros dias de incubação. Isto era esperado mesmo para tratamentos que não receberam matéria orgânica (controle e FR) pois, o manuseio das amostras e o seu umedecimento são estímulos para a comunidade microbiana decompor compostos orgânicos próprios da amostra de solo utilizada. Para estes tratamentos foram registrados, nesta fase inicial, cerca de $37 \%$ do total de carbono liberado. A adição de poaia ao solo, com ou sem adição de $\mathrm{N}$ e/ou FR, resultou em aumentos na liberação de carbono do sistema sendo que, cerca de $77 \%$ do carbono total liberado foram registrados nos primeiros dez dias de incubação, com valores semelhantes aos encontrados para solo com incorporação de palha de milho, mas inferiores àqueles observados em solo adicionado de vinhaça e bagaço de cana (Minhoni et al., 1990). Este comportamento observado para a liberação de carbono é frequiente na literatura e é um reflexo da sucessão de comunidades e metabolismos microbianos no sistema. Inicialmente, a maior quantidade de compostos solúveis, prontamente decomponíveis, promovem aumentos na comunidade microbiana, em termos de metabolismo e população; após, os compostos restantes mais complexos, determinam uma comunidade microbiana diferente e menor, de acordo com os dados de liberação de $\mathrm{CO}_{2}$ e formação de biomassa microbiana do presente experimento (Tabela 1 e Figura 1).

$\mathrm{O}$ nitrogênio parece não ter se constituído em fator limitante do metabolismo microbiano em termos de $\mathrm{CO}_{2}$ liberado, tanto que nos cinco primeiros dias, este parâmetro foi maior nos tratamentos sem adição do mesmo, ou seja, poaia e poaia+FR. Contudo, à partir do sétimo dia, a liberação de $\mathrm{CO}_{2}$ nos tratamentos com nitrogênio $($ poaia $+\mathrm{N}$ e poaia $+\mathrm{N}+\mathrm{FR})$ foi igual ou maior aos tratamentos sem nitrogênio. Se for considerado o dado médio de $\mathrm{CO}_{2}$ liberado nos tratamentos com poaia e controle, para todo o período de incubação, a liberação de $\mathrm{CO}_{2}$ foi 57 vezes maior nos tratamentos com poaia. Durante a incubação, o tratamento poaia+N+FR apresentou tendência de maiores valores de $\mathrm{CO}_{2}$ liberado. Eira \& Minhoni (1991) referem que a adição de matéria orgânica e nutrientes ao solo, principalmente nitrogênio e fósforo, promove aumento da comunidade microbiana. Minhoni et al. (1990), também concluíram que a adição simultânea de nitrogênio e fósforo, em solo adicionado de diferentes tipos de matéria orgânica, aumenta significativamente as taxas respiratórias da decomposição de vinhaça, glicose e bagaço de cana.

Quanto à biomassa microbiana, os valores foram constantes e geralmente iguais nos tratamentos controle e FR, durante todo o período de incubação (Figura 1). A adição de poaia provocou aumentos na biomassa, com valores menores àqueles encontrados por Minhoni et al. (1996) em solo com adição de glicose, porém maiores aos encontrados por Minhoni \& Cerri (1987) em solo adicionado de vinhaça. Na presença de nitrogênio e/ou fosfato de rocha, os picos de biomassa ocorreram aos dez dias de incubação enquanto que no tratamento com poaia apenas, aos vinte dias de incubação. Contrariamente ao observado para o parâmetro anterior, estes resultados são evidências de que o nitrogênio e o fósforo, isolados ou em conjunto, tenham sido fatores limitantes na formação de biomassa microbiana. Desprezando-se o carbono da amostra de terra, os valores máximos de biomassa microbiana são muito próximos entre si e correspondem à cerca de $7 \%$ do carbono adicionado como poaia. Entretanto, ao final do período de incubação, a biomassa microbiana foi igual para todos os tratamentos com poaia, e o seu dado médio foi cerca de oito vezes maior à biomassa do tratamento controle. Ainda na Figura 1, observa-se ausência de dados iniciais de biomassa microbiana nos tratamentos adicionados de poaia. Este comportamento é comum na literatura e tem sido atribuído ao fato de que em parcelas fumigadas existe uma incapacidade da população reinoculada em degradar o substrato 
TABELA 1. Taxa diária média de carbono liberado do solo $\left(\mathrm{mg} \mathrm{C}^{\mathrm{kg}}{ }^{-1} \mathrm{solo}\right)$ de parcelas não fumigadas, em função da adição de matéria seca de Richardia brasiliensis, nitrogênio e/ou fosfato de rocha, Botucatu, 1992.

\begin{tabular}{lcccccccccccccc}
\hline & \multicolumn{10}{c}{ Incubação (dias) } \\
\cline { 2 - 5 }$(1)$ & 2 & 5 & 7 & 10 & 15 & 20 & 25 & 30 & 35 & 40 & 45 & 50 & 55 & 60 \\
\hline Controle & $3,6 \mathrm{c}^{(2)}$ & $4,7 \mathrm{c}$ & $5,0 \mathrm{c}$ & $4,1 \mathrm{c}$ & $4,0 \mathrm{~b}$ & $2,9 \mathrm{c}$ & $4,0 \mathrm{c}$ & $3,0 \mathrm{c}$ & $2,1 \mathrm{~b}$ & $2,0 \mathrm{c}$ & $4,5 \mathrm{~b}$ & $1,9 \mathrm{~b}$ & $2,5 \mathrm{~b}$ & $3,4 \mathrm{~b}$ \\
Poaia & $463 \mathrm{~b}$ & $729 \mathrm{a}$ & $572 \mathrm{~b}$ & $309 \mathrm{~b}$ & $153 \mathrm{a}$ & $89 \mathrm{ab}$ & $67 \mathrm{~b}$ & $57 \mathrm{~b}$ & $49 \mathrm{a}$ & $49 \mathrm{ab}$ & $44 \mathrm{a}$ & $34 \mathrm{a}$ & $27 \mathrm{a}$ & $19 \mathrm{a}$ \\
Poaia + N & $406 \mathrm{~b}$ & $615 \mathrm{~b}$ & $741 \mathrm{a}$ & $404 \mathrm{a}$ & $159 \mathrm{a}$ & $91 \mathrm{ab}$ & $80 \mathrm{~b}$ & $87 \mathrm{a}$ & $54 \mathrm{a}$ & $61 \mathrm{ab}$ & $54 \mathrm{a}$ & $37 \mathrm{a}$ & $28 \mathrm{a}$ & $17 \mathrm{a}$ \\
Poaia+N+FR & $446 \mathrm{~b}$ & $603 \mathrm{~b}$ & $764 \mathrm{a}$ & $408 \mathrm{a}$ & $159 \mathrm{a}$ & $102 \mathrm{a}$ & $102 \mathrm{a}$ & $94 \mathrm{a}$ & $60 \mathrm{a}$ & $64 \mathrm{a}$ & $56 \mathrm{a}$ & $36 \mathrm{a}$ & $25 \mathrm{a}$ & $25 \mathrm{a}$ \\
Poaia + FR & $541 \mathrm{a}$ & $719 \mathrm{a}$ & $569 \mathrm{~b}$ & $308 \mathrm{~b}$ & $147 \mathrm{a}$ & $86 \mathrm{~b}$ & $69 \mathrm{~b}$ & $70 \mathrm{ab}$ & $49 \mathrm{a}$ & $46 \mathrm{~b}$ & $46 \mathrm{a}$ & $39 \mathrm{a}$ & $29 \mathrm{a}$ & $21 \mathrm{a}$ \\
FR & $3,6 \mathrm{c}$ & $1,3 \mathrm{~d}$ & $7,3 \mathrm{c}$ & $4,1 \mathrm{c}$ & $2,6 \mathrm{~b}$ & $2,9 \mathrm{c}$ & $1,5 \mathrm{c}$ & $2,0 \mathrm{c}$ & $2,1 \mathrm{~b}$ & $3,9 \mathrm{c}$ & $4,9 \mathrm{~b}$ & $1,4 \mathrm{~b}$ & $2,5 \mathrm{~b}$ & $3,3 \mathrm{~b}$ \\
\hline C.V. & 4,8 & 2,1 & 3,4 & 2,3 & 2,7 & 4,6 & 7,1 & 11,2 & 10,1 & 8,1 & 13,1 & 17,2 & 10,6 & 15,9 \\
\hline
\end{tabular}

(1) Tratamentos:controle (sem adição de poaia,nitrogênio e fosfato de rocha); poaia (apenas poaia); poaia $+\mathrm{N}$ (poaia+nitrogênio); poaia $+\mathrm{N}+$

FR (poaia+nitrogênio + fosfato de rocha); poaia + FR (poaia + fosfato de rocha); FR (apenas fosfato de rocha).

(2) Para cada período de incubação, médias seguidas de letras iguais não diferem significativamente entre si, de acordo com o teste de Tukey a $5 \%$ de significância.

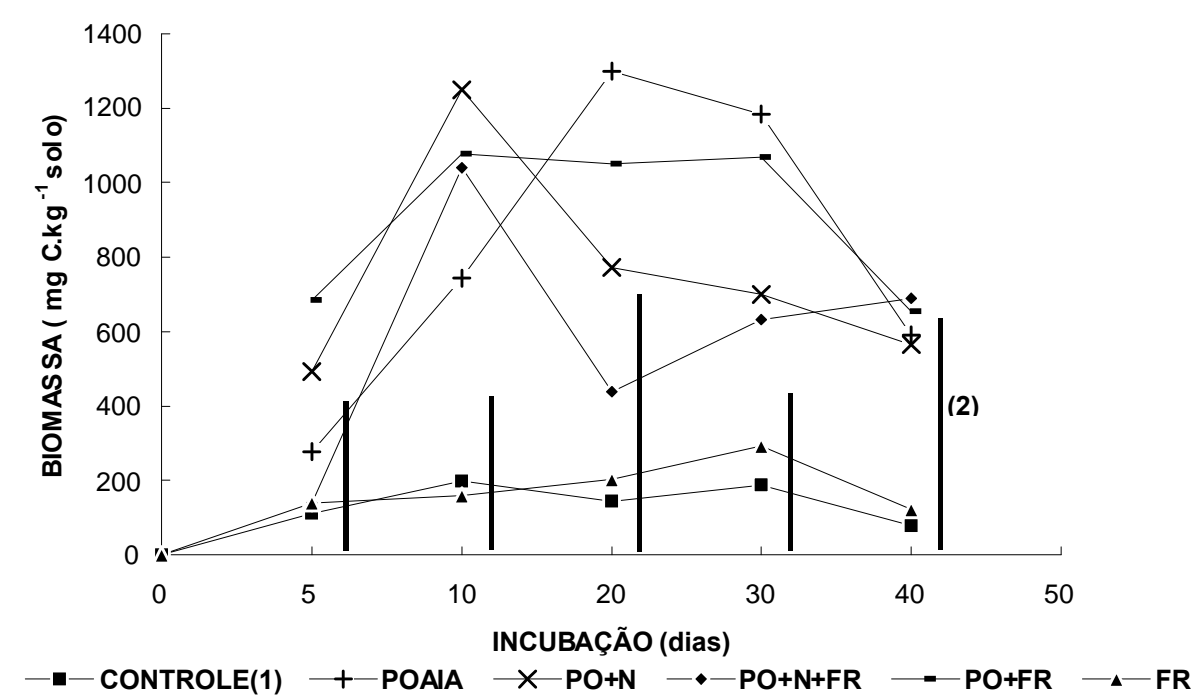

FIGURA 1. Valores médios de biomassa microbiana ( $\mathrm{mg} \mathrm{C} \mathrm{kg}^{-1}$ solo), em função da adição de matéria seca de Richardia brasiliensis, nitrogênio e/ou fosfato de rocha a uma Areia Quartzosa, Botucatu, 1992.

(1) Tratamentos: controle (sem adição de poaia, nitrogênio e/ou fosfato de rocha); poaia (apenas poaia); poaia $+\mathrm{N}$ (poaia + nitrogênio); poaia+N+FR (poaia + nitrogênio + fosfato de rocha); poaia+FR (poaia + fosfato de rocha); FR (apenas fosfato de rocha).

(2) DMS à 5\% para cada período de incubação. 
tão rapidamente quanto em parcelas não fumigadas, resultando em uma inversão na liberação de $\mathrm{CO}_{2}$, ou seja, parcelas fumigadas liberam menos $\mathrm{CO}_{2}$ que as não fumigadas (Jenkinson \& Powlson, 1976) (Figura 2).
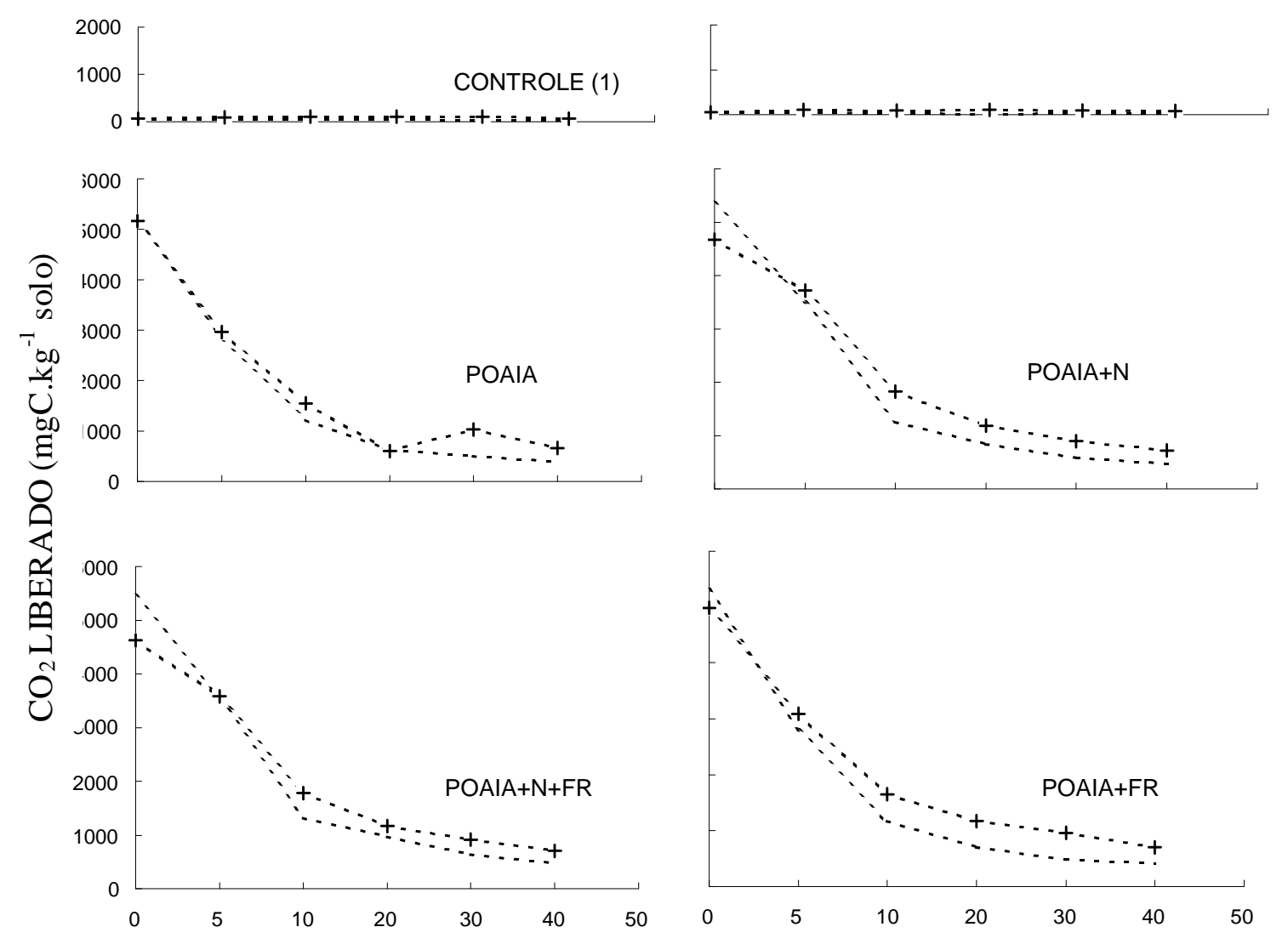

FIGURA 2. Valores médios de $\mathrm{CO}_{2}$ liberado (mgC. $\mathrm{kg}^{-1}$ solo) de tratamentos fumigados (--+--) e não fumigados (-----), em função da adição de matéria seca de Richardia brasiliensis, nitrogênio e/ou fosfato de rocha a uma Areia Quartzosa, Botucatu, 1992.

(1) Tratamentos: controle (sem adição de poaia, nitrogênio e/ou fosfato de rocha); poaia (apenas poaia); poaia $+\mathrm{N}$ (poaia+nitrogênio); poaia+N+FR (poaia+nitrogênio+fosfato de rocha); poaia+FR (poaia+fosfato de rocha); FR (apenas fosfato de rocha).

A Tabela 2 apresenta os dados médios de fósforo disponível. Nos tratamentos controle e FR os teores mantiveram-se ao redor de 7 e $171 \mathrm{mg}$ de $\mathrm{P} \mathrm{kg}^{-1}$, respectivamente, durante todo o período de incubação. Para os tratamentos adicionados de poaia, sem fosfato de rocha (poaia e poaia $+\mathrm{N}$ ), os dados de fósforo disponível foram geralmente iguais entre si, com média de cerca de 38mg de $\mathrm{P} \mathrm{kg}{ }^{-1}$, durante todo o período de incubação. A presença de fosfato de rocha junto à poaia, com e sem nitrogênio, aumentou os teores médios de fósforo disponível para 76 e $90 \mathrm{mg}$ de $\mathrm{P} \mathrm{kg}^{-1}$, respectivamente, porém, não foi suficiente para superar os dados observados no tratamento com fosfato de rocha apenas. 
TABELA 2. Teores médios de fósforo disponível ( $\left.\mathrm{mg} \mathrm{de} \mathrm{P} \mathrm{kg}^{-1}\right)$, obtidos durante o período de incubação do solo, na presença de matéria seca de Richardia brasiliensis, nitrogênio e/ou fosfato de rocha, Botucatu, 1992.

\begin{tabular}{|c|c|c|c|c|c|c|c|c|c|c|c|}
\hline \multirow{2}{*}{ Tratamentos $^{(1)}$} & \multicolumn{11}{|c|}{ Incubação (dias) } \\
\hline & 0 & 2 & 3 & 5 & 7 & 10 & 20 & 30 & 40 & 50 & 60 \\
\hline Controle & $6 \mathrm{e}^{(2)}$ & $7 e$ & $7 e$ & $7 \mathrm{~d}$ & $7 d$ & $7 \mathrm{e}$ & $8 \mathrm{~d}$ & $7 \mathrm{e}$ & $7 \mathrm{e}$ & $7 f$ & $7 f$ \\
\hline Poaia & $44 d$ & $42 \mathrm{~d}$ & $40 \mathrm{~d}$ & $31 \mathrm{c}$ & $30 c$ & $28 \mathrm{~d}$ & $41 \mathrm{c}$ & $44 \mathrm{~cd}$ & $50 \mathrm{c}$ & $51 \mathrm{~d}$ & $53 d$ \\
\hline Poaia+N & $37 d$ & $37 d$ & $43 \mathrm{~d}$ & $36 \mathrm{c}$ & $25 \mathrm{c}$ & $25 \mathrm{~d}$ & $44 c$ & $36 \mathrm{~d}$ & $37 d$ & $37 \mathrm{e}$ & $37 \mathrm{e}$ \\
\hline Poaia+N+FR & $89 \mathrm{c}$ & $91 \mathrm{c}$ & $87 \mathrm{c}$ & $88 \mathrm{~b}$ & $71 b$ & $65 c$ & $66 b$ & $65 c$ & $78 b$ & $70 \mathrm{c}$ & $69 c$ \\
\hline Poaia+FR & $107 \mathrm{~b}$ & $118 b$ & $112 b$ & $81 b$ & $72 b$ & $77 \mathrm{~b}$ & $80 \mathrm{~b}$ & $87 \mathrm{~b}$ & $84 b$ & $85 b$ & $90 \mathrm{~b}$ \\
\hline FR & $149 \mathrm{a}$ & $180 \mathrm{a}$ & $174 a$ & $168 \mathrm{a}$ & $178 \mathrm{a}$ & $192 \mathrm{a}$ & $166 a$ & $162 \mathrm{a}$ & $166 \mathrm{a}$ & $180 \mathrm{a}$ & $166 \mathrm{a}$ \\
\hline C.V. (\%) & 9 & 11 & 11 & 8 & 12 & 8 & 14 & 14 & 8 & 4 & 5 \\
\hline
\end{tabular}

Com relação ao $\mathrm{pH}$, este parâmetro foi menor no tratamento controle durante todo o período de incubação, com valor médio de 4,3 (Tabela 3). A adição de fosfato de rocha apenas (FR) fez com que o dado médio se elevasse para 4,9 , provavelmente devido à adsorção específica do fosfato às partículas de óxido de ferro do solo, liberando radicais hidroxila (Minhoni et al., 1996). Por outro lado, a presença de poaia, sem adição de nitrogênio (poaia e poaia+FR), resultou em alcalinização do sistema, com variações no $\mathrm{pH}$ de 5,5 a 7,6. Esta alcalinização também foi verificada por Minhoni et al. (1991), quando da adição de algumas fontes de carbono ao solo, como a palha de soja e vinhaça. A presença de nitrogênio junto à poaia, com ou sem fosfato de rocha, modificou a resposta, ou seja, o sistema manteve-se ácido, variando de 5,1 a 5,7. Infere-se que nestes tratamentos a produção de ácidos orgânicos deva ter sido incrementada pela adição do nitrogênio, reduzindo o efeito alcalinizante da poaia. Estes menores valores de $\mathrm{pH}$ nos tratamentos adicionados de nitrogênio podem também estar relacionados à nitrificação do amônio, a qual resulta em formação de íons $\mathrm{H}^{+}$ (Victoria et al., 1992).

Muitos autores já observaram que o aumento de fósforo disponível, em decorrência da solubilização microbiana de fosfatos, está relacionado a um abaixamento do $\mathrm{pH}$ do sistema (Sperber, 1958; Minhoni et al., 1996 e Ortuño et al., 1978ab), havendo, desta forma, correlação inversa entre os dois parâmetros. Nahas et al. (1994) encontraram um outro tipo de relação; aumentos no $\mathrm{pH}$ até um valor ótimo para o crescimento microbiano, coincidiram com aumentos nos valores de fósforo disponível. No presente trabalho, apesar da alcalinização do sistema em função da adição de matéria seca de poaia, não se pode afirmar que não houve solubilização do fosfato incorporado, pois a comunidade microbiana aumentada, em termos de liberação de $\mathrm{CO}_{2}$ e formação de biomassa microbiana, pode ter utilizado o fósforo solubilizado para seu metabolismo. 
TABELA 3. pH médios, obtidos durante o período de incubação do solo, na presença de matéria seca de Richardia brasiliensis, nitrogênio e/ou fosfato de rocha, Botucatu, 1992.

\begin{tabular}{|c|c|c|c|c|c|c|c|c|c|c|c|}
\hline \multirow{2}{*}{ Tratamentos $^{(1)}$} & \multicolumn{11}{|c|}{ Incubação (dias) } \\
\hline & 0 & 2 & 3 & 5 & 7 & 10 & 20 & 30 & 40 & 50 & 60 \\
\hline Controle & $4,2 d^{(2)}$ & $4,1 \mathrm{~d}$ & $4,1 \mathrm{~d}$ & $4,1 \mathrm{e}$ & $4,1 d$ & $4,2 d$ & $4,4 d$ & $4,7 \mathrm{~d}$ & $4,5 \mathrm{~d}$ & $4,5 \mathrm{~d}$ & $4,3 \mathrm{~d}$ \\
\hline Poaia & $5,5 \mathrm{a}$ & $5,7 \mathrm{a}$ & $5,8 \mathrm{a}$ & $6,4 \mathrm{a}$ & $6,7 \mathrm{a}$ & $6,8 \mathrm{a}$ & $7,3 \mathrm{a}$ & $7,5 \mathrm{a}$ & $7,5 \mathrm{a}$ & $7,6 a$ & $7,6 \mathrm{a}$ \\
\hline Poaia+N & $5,2 b$ & $5,1 b$ & $5,1 \mathrm{~b}$ & $5,2 \mathrm{c}$ & $5,2 b$ & $5,1 \mathrm{~b}$ & $5,3 b$ & $5,7 \mathrm{~b}$ & $5,2 \mathrm{c}$ & $5,2 \mathrm{~b}$ & $5,4 b$ \\
\hline Poaia+N+FR & $5,2 \mathrm{~b}$ & $5,2 \mathrm{~b}$ & $5,1 \mathrm{~b}$ & $5,3 \mathrm{~b}$ & $5,3 \mathrm{~b}$ & $5,1 b$ & $5,4 \mathrm{~b}$ & $5,7 \mathrm{~b}$ & $5,2 \mathrm{c}$ & $5,2 \mathrm{~b}$ & $5,5 \mathrm{~b}$ \\
\hline Poaia+FR & $5,5 \mathrm{a}$ & $5,9 \mathrm{a}$ & $6,0 \mathrm{a}$ & $6,4 a$ & $6,8 \mathrm{a}$ & $6,8 \mathrm{a}$ & $7,3 \mathrm{a}$ & $7,4 \mathrm{a}$ & $7,6 \mathrm{a}$ & $7,6 \mathrm{a}$ & $7,6 \mathrm{a}$ \\
\hline FR & $4,6 \mathrm{c}$ & $4,6 c$ & $4,6 c$ & $4,7 d$ & $4,8 \mathrm{c}$ & $4,8 \mathrm{a}$ & $5,2 \mathrm{c}$ & $5,3 \mathrm{c}$ & $5,1 \mathrm{c}$ & $5,0 \mathrm{c}$ & $4,9 \mathrm{c}$ \\
\hline C.V. (\%) & 1,8 & 2,6 & 2,1 & 0,6 & 1,3 & 0,9 & 1,1 & 0,9 & 0,6 & 1,1 & 0,9 \\
\hline
\end{tabular}

Tratamentos: controle (sem adição de poaia, nitrogênio e/ou fosfato de rocha); poaia (apenas poaia); poaia $+\mathrm{N}$ (poaia + nitrogênio); poaia+N+FR (poaia + nitrogênio + fosfato de rocha); poaia+FR (poaia + fosfato de rocha) e FR (apenas fosfato de rocha).

(2) Para cada período de incubação, médias seguidas de letras iguais, não diferem entre si, de acordo com o teste de Tukey, à 5\% de significância.

\section{LITERATURA CITADA}

ALVES, P.L.C.A. Interações alelopáticas entre plantas daninhas e hortaliças. In: SIMPÓSIO NACIONAL SOBRE MANEJO INTEGRADO DE PLANTAS DANINHAS EM HORTALIÇAS, 1, 1992, Botucatu. Botucatu: Faculdade de Ciências Agronômicas, 1992. p.19-43.

BONDE, T.A., SCHNURER, J., ROSSWAL, T. Microbial biomass as fraction of potentially mineralizable nitrogen in soils from longterm field experiments. Soil Biol. Biochem., v.20, p.447-452, 1989.

COUTEAUX, M.M., HENKINET, R., PITTA, P., et al. Native carbon mineralization of an acid organic soil after use of the chloroform-fumigation method to estimate microbiol biomass. Biol. Fert. Soils, v.8, p.172-177, 1989.
EIRA, A.F. Solubilização microbiana de fosfatos. In: CARDOSO, E.J.B.N., TSAI, S.M., NEVES, M.C.P. (Eds.) Microbiologia do solo. Campinas: Sociedade Brasileira de Ciência do Solo, 1992. Cap. 17, p.243-255.

EIRA, A.F., MINHONI, M.T.A. Manual prático de biotecnologia e microbiologia agrícola. 3- Microbiologia do solo (a população microbiana do solo). Botucatu: FEPAF, 1991. 45p.

JENKINSON, D.S., POWLSON, D.S. The effects of biocidal treatments on metabolism in soil. V - A method for measuring soil biomass. Soil Biol. Biochem., v.8, p.209213, 1976.

MINHONI, M.T.A., CERRI, C.C. Decomposição de vinhaça em solo sob diferentes níveis de umidade: liberação de $\mathrm{CO}_{2}$, formação de biomassa microbiana e imobilização do nitrogênio adicionado. R. Bras. Ci. Solo, v.11, p.25-30, 1987. 
MINHONI, M.T.A., EIRA, A.F., CARDOSO, E.J.B.N. Efeitos da adição de $\mathrm{N}$ e $\mathrm{P}$ sobre a decomposição de diferentes tipos de material orgânico no solo. R. Bras. Ci. Solo, v.14, p.297-304, 1990.

MINHONI, M.T.A., CARDOSO, E.J.B.N., EIRA, A.F. Efeito de cinco tipos de matéria orgânica na solubilização microbiana de fosfato de rocha. R. Bras. Ci. Solo, v.15, p. 29-35, 1991.

MINHONI, M. T. A., EIRA, A. F., BÜLL, L. T. Biomassa microbiana, liberação de $\mathrm{CO}_{2}$, fósforo disponível e $\mathrm{pH}$ em solo que recebeu glicose e fosfato de rocha. R. Bras. Ci. Solo, v. 20, p. 387-392, 1996.

NAHAS, E., CENTURION, J.F., ASSIS, L.C. Efeito das características químicas dos solos sobre os microrganismos solubilizadores de fosfato e produtores de fosfatases. R. Bras. Ci. Solo, v.18, p. 49-53, 1994.

ORTUÑO, A., HERNANSAÉS, A., NOGUEIRA, J., et al. Acción solubilizadora del fósforo por Aspergillus niger e Pseudomonas fluorescens. Microbiol. Esp., v.30-31, p.113-120, 1978a.

ORTUÑO, A., HERNANSAÉS, A., NOGUEIRA, $\mathrm{J}$, et al. Metabolismo fosfórico de Aspergillus niger em suelos calizos $\mathrm{y}$ salinos. Microbiol. Esp., v.30-31, p.101-
112, 1978b.

RAIJ, B.V., QUAGGIO, J.A. Métodos de análise de solo para fins de fertilidade. Boletim Técnico IAC, n.81, 31p., 1983.

SAFFIGNA, P.G., POWLSON, D.S., BROOKS, P.C., THOMAS, G.A. Influence of sorghum residues and tillage on soil organic matter and soil microbial biomass in an australian vertisol. Soil Biol. Biochem., v.21, p.759-765, 1989.

SALVATI, S.S. Estudos sobre interações alelopáticas envolvendo plantas daninhas e cultivadas. Botucatu, $1988 . \quad 45 \mathrm{p}$. Dissertação (Licenciatura em Ciências Biológicas) - Instituto de Biociências, Universidade Estadual Paulista.

SNEDECOR, G.W.E., COCHRAN, W.G. Statistical methods. The Iwoa State Univ.

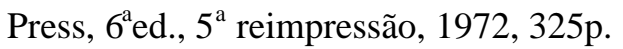

SPERBER, J.I. The incidence of apatite solibilizing organisms in the rhizosphere and soil. Aust. J. Agric. Res., v.9, p.778$787,1958$.

VICTORIA, R. L., PICCOLO, M. C., VARGAS, A. A. T. O ciclo do nitrogênio. In: CARDOSO, E.J.B.N., TSAI, S.M., NEVES, M.C.P. (Eds). Microbiologia do solo. Campinas: Sociedade Brasileira de Ciência do Solo, 1992. Cap.8, p.105-119. 